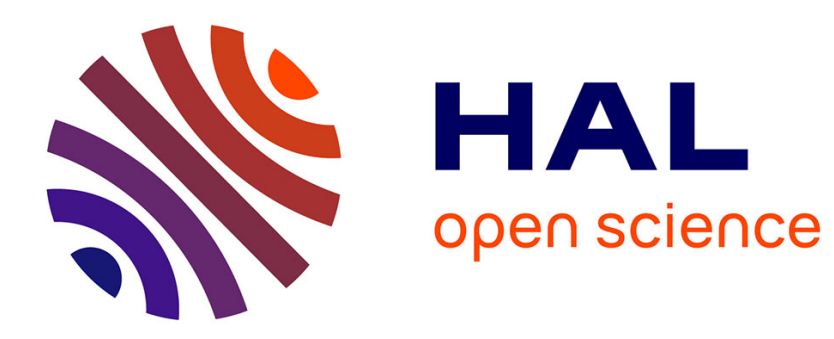

\title{
On waveguide modeling of stiff piano string
}

Eric Ducasse

\section{- To cite this version:}

Eric Ducasse. On waveguide modeling of stiff piano string. Journal of the Acoustical Society of America, 2005, 118 (3), pp.1776-1781. 10.1121/1.1993127 . hal-00869870

\section{HAL Id: hal-00869870 https://hal.science/hal-00869870}

Submitted on 4 Oct 2013

HAL is a multi-disciplinary open access archive for the deposit and dissemination of scientific research documents, whether they are published or not. The documents may come from teaching and research institutions in France or abroad, or from public or private research centers.
L'archive ouverte pluridisciplinaire HAL, est destinée au dépôt et à la diffusion de documents scientifiques de niveau recherche, publiés ou non, émanant des établissements d'enseignement et de recherche français ou étrangers, des laboratoires publics ou privés. 


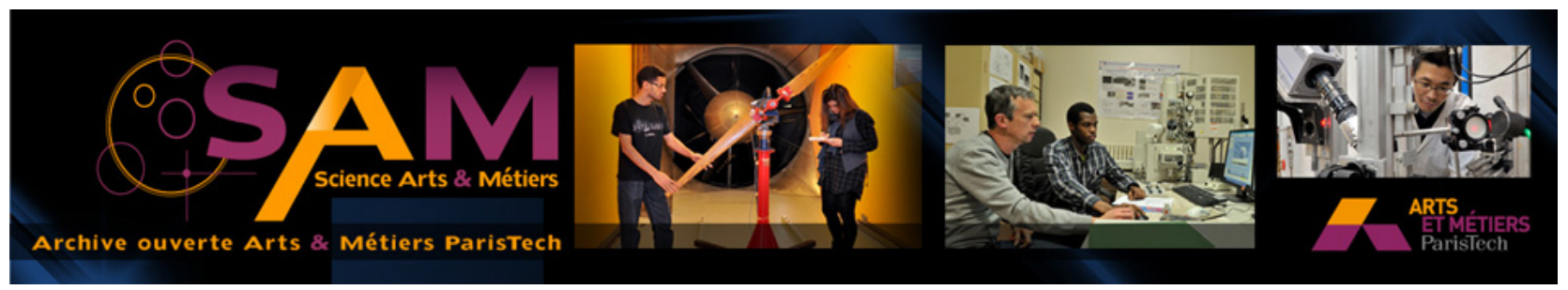

Science Arts \& Métiers (SAM)

is an open access repository that collects the work of Arts et Métiers ParisTech researchers and makes it freely available over the web where possible.

This is an author-deposited version published in: http://sam.ensam.eu

Handle ID: .http://hdl.handle.net/10985/7362

\section{To cite this version :}

Eric DUCASSE - On waveguide modeling of stiff piano string - J. Acoust. Soc. Am. - Vol. 118, $\mathrm{n}^{\circ} 3$, p.1776-1781 - 2005 


\title{
On waveguide modeling of stiff piano strings
}

\author{
Éric Ducasse
}

École Nationale Supérieure d'Arts et Métiers, C.E.R. de Bordeaux-Talence, 33405 Talence cedex, France

eric.ducasse@ensam.eu

\section{ABSTRACT}

Bensa et al. [J. Acoust. Soc. Am. 114(2) 1095-1107 (2003), Section IV] recently proposed a waveguide model for the transverse displacement of a stiff piano string. The study described here is an attempt to cast a complementary light on this topic, based on a common wave approach instead of a modal approach. A pair of weakly attenuated traveling waves and a pair of fast-decaying waves both satisfy the one-dimensional wave equation developed by Bensa et al.. These solutions have to be carefully considered, however, for portions of string interacting with the hammer felt, the bridge, or the capo d'astro bar.

PACS number: 43.75.Mn

\section{INTRODUCTION}

The first attempt for synthesizing musical sounds using physical models was made more than 30 years ago by Hiller and Ruiz ${ }^{1}$. Starting from the one-dimensional wave equation ${ }^{2}$ of the transverse displacement of a string, three main approaches are generally used for building a computational model: either the use of a finite difference scheme (e.g. Refs. 3, 4), or a modal synthesis (e.g. Ref. 5), or the building of a Digital Waveguide model (e.g. Refs. 6, 7,3). A waveguide is considered here as a "filterlike structure modeling one-dimensional wave propagation as purely lossless throughout the length of the string, with loss and dispersion lumped in terminating filters ${ }^{3}$." The hammer action and the reflection conditions at both ends of the string are also modeled as lumped filters. Portions of string are distributed elements ${ }^{6}$ represented by two-port networks. In this paper we focus on this latter approach.

Bensa et al. propose an improved one-dimensional wave equation [Ref. 3, Eq. (6)] corresponding to a well posed model of stiff piano strings. In Sec. IV.B they use a modal approach to extract the waveguide parameters from the partial differential equation (PDE). This approach requires the knowledge of both the length of the string and the boundary conditions [Ref. 3, Eq. (10)] for the calculation of the vibration modes associated with standing waves. The waveguide parameters are then deduced from the characteristics of the first mode including the fundamental frequency of the ideal string. The present paper is an attempt to show that a wave approach using complex wave-numbers appears as more convenient because each dispersive attenuated traveling wave is directly obtained from the one-dimensional wave equation, apart from the reflections at the ends of the string. Assuming that the one-dimensional wave equation is linear, time- and space-invariant, a two-dimensional Fourier-Laplace transformation gives an algebraic equation relating the transverse displacement of the string (output) to the space distribution of external forces (input) in the wave-number/frequency domain. The solution of this equation can be seen as the superposition of traveling waves which occur in a waveguide model.

Some general points about waveguides, traveling waves, and complex wave-numbers are outlined in Section II to help the reader to understand the following sections. In Section III, after the one-dimensional wave equation advanced by Bensa et $a .^{3}$ is recalled, this equation is solved to obtain the response of an infinite string to a point impulse in the wave-number/frequency domain, showing that two fast-decaying traveling waves are omitted by the modal approach in Bensa et al. ${ }^{3}$. These fast-decaying waves can be neglected only for portions of string without sources which are long enough. They should, however, be taken into account at the neighborhood of the excitation point, as detailed in Section IV. 


\section{WAVEGUIDE MODELING AND COMPLEX WAVE-NUMBERS}

The question is this: how to pass from the wave equation of a one-dimensional system to a waveguide model?

\section{A. From the one-dimensional wave equation to the transfer function}

\section{A general one-dimensional wave equation}

The vibration state of a one-dimensional system (e.g. the stiff piano string) is assumed to be defined by a function $y$ (e.g. the transverse displacement of the string) of time $t$ and position $x$ along the system.

A general one-dimensional wave equation, assuming that it is linear, time- and space-invariant, can be written as:

$$
\sum_{m=0}^{d_{x}} \sum_{n=0}^{d_{t}} \alpha_{m, n} \frac{\partial^{m+n} y}{\partial x^{m} \partial t^{n}}(x, t)=f(x, t)
$$

where $f$ is the space distribution of external forces (e.g. exerted by the hammer felt, the bridge and the capo d'astro bar).

\section{Time/frequency and space/wave-number conversions}

In the space/frequency domain, each signal $s(x, t)$ is converted into $\widehat{s}(x, \omega)$ by Fourier transformation:

$$
\widehat{s}(x, \omega)=\int_{-\infty}^{+\infty} s(x, t) e^{-i \omega t} d t
$$

where $\omega$ is the angular frequency and $i^{2}=-1$.

In the wave-number/frequency domain, each signal $s(x, t)$ becomes $S(k, \omega)$ by Fourier-Laplace transformation:

$$
S(k, \omega)=\int_{-\infty}^{+\infty} \widehat{s}(x, \omega) e^{-i k x} d x=\int_{-\infty}^{+\infty} \int_{-\infty}^{+\infty} s(x, t) e^{-i(\omega t+k x)} d t d x
$$

where $k$ is the complex wave-number.

\section{An ordinary differential equation in the space/frequency domain}

The Fourier transformation of the wave equation (1) leads to an ordinary linear constant-coefficient differential equation with respect to $x$ :

$$
\sum_{m=0}^{d_{x}}\left[\sum_{n=0}^{d_{t}} \alpha_{m, n}(i \omega)^{n}\right] \frac{\partial^{m} \widehat{y}}{\partial x^{m}}(x, \omega)=\widehat{f}(x, \omega)
$$

\section{The transfer function}

In the wave-number/frequency domain, we obtain:

$$
\left[\sum_{m=0}^{d_{x}} \sum_{n=0}^{d_{t}} \alpha_{m, n} i^{m+n} \omega^{n} k^{m}\right] Y(k, \omega)=F(k, \omega) \Longleftrightarrow Y(k, \omega)=H(k, \omega) F(k, \omega),
$$

where $H(k, \omega)$ is the transfer function of the system. The denominator of $H(k, \omega)$ is a polynomial of order $d_{x}$ in $k$ and order $d_{t}$ in $\omega$. The poles of $H(k, \omega)$ (where $H(k, \omega)$ is considered as a function of $k$ ) are the roots of a polynomial equation commonly called the dispersion equation. 


\section{B. From the transfer function to the block-diagram model}

\section{The impulse response as the sum of pairs of symmetrical traveling waves}

Assuming both that the propagation model corresponds to a well-posed physical problem (cf. e.g. Ref. 3 for more details) and that the problem is symmetrical (i.e., unchanged by substituting $-x$ for $x$ ), the coefficients $\alpha_{2 k+1, n}$ are zero, which implies that $d_{x}=2 J$ is an even number, and the transfer function $H(k, \omega)$ is rewritten as follows:

$$
H(k, \omega)=\frac{a(\omega)}{\prod_{j=1}^{J}\left[k^{2}-k_{j}(\omega)^{2}\right]}=\sum_{j=1}^{J} \frac{a_{j}(\omega)}{k^{2}-k_{j}(\omega)^{2}}=\sum_{j=1}^{J} g_{j}(\omega)\left[\frac{1}{k-k_{j}(\omega)}-\frac{1}{k+k_{j}(\omega)}\right]
$$

where $a_{j}(\omega)=a(\omega) / \prod_{m=1, m \neq j}^{J}\left[k_{m}(\omega)^{2}-k_{j}(\omega)^{2}\right]$ and $g_{j}(\omega)=a_{j}(\omega) /\left[2 k_{j}(\omega)\right]$, assuming $k_{j}(\omega) \neq 0$ and $k_{j}(\omega) \neq k_{m}(\omega)$ if $j \neq m$.

For an infinite system, the assumed boundary (no sources at infinity) and initial conditions (rest initial condition) are:

$$
\left.y\right|_{x \rightarrow+\infty}=\left.y\right|_{x \rightarrow-\infty}=0 ;\left.y\right|_{t<0}=0 ; \forall n, 1 \leqslant n \leqslant d_{t}-1,\left.\frac{\partial^{n} y}{\partial t^{n}}\right|_{t<0}=0 .
$$

Coming back to the space/frequency domain, Eqs. (6) and (7) lead to the convolution product with respect to the spatial variable:

$$
\widehat{y}(x, \omega)=\int_{-\infty}^{+\infty} \widehat{f}\left(x_{0}, \omega\right) \widehat{h}\left(x-x_{0}, \omega\right) d x_{0}
$$

$\widehat{h}(x, \omega)$ satisfying

$$
\widehat{h}(x, \omega)=\sum_{j=1}^{J} g_{j}(\omega)\left[e^{-i k_{j}(\omega) x} u(x)+e^{+i k_{j}(\omega) x} u(-x)\right],
$$

where $u$ is the Heaviside unit step function:

$$
\forall x<0, u(x)=0 \text { and } \forall x \geqslant 0, u(x)=1 .
$$

The function $\widehat{h}(x, \omega)$ corresponds to the Fourier transform of the impulse response $h(x, t)$ (also named Green's function) to $f(x, t)=\delta(x) \delta(t)$ where $\delta$ is the Dirac impulse. This response $h(x, t)$ is the effect of a time impulse (at $t=0$ ) exerted on a single point (at $x=0$ ) of the system. The unit of $h$ is the unit of $y$ per Newton and per second. An inverse Fourier transformation gives:

$$
h(x, t)=\frac{1}{2 \pi} \sum_{j=1}^{J} \int_{-\infty}^{+\infty} g_{j}(\omega)\left\{e^{i\left[\omega t-k_{j}(\omega) x\right]} u(x)+e^{i\left[\omega t+k_{j}(\omega) x\right]} u(-x)\right\} d \omega .
$$

Note that $h(x, t)=h(-x, t)$ : the symmetry of the problem is satisfied.

Each $k_{j}(\omega)$ is a complex wave-number such that:

$$
k_{j}(\omega)=\omega \tau_{j}(\omega)-i \alpha_{j}(\omega)=\frac{\omega}{c_{j}(\omega)}-i \alpha_{j}(\omega)
$$

where $\tau_{j}(\omega) \geqslant 0$ is the propagation delay per unit length (or slowness), $c_{j}(\omega)$ the phase velocity, and $\alpha_{j}(\omega) \geqslant 0$ the attenuation per unit length.

The impulse response can be rewritten as:

$$
h(x, t)=\frac{1}{2 \pi} \sum_{j=1}^{J} \int_{-\infty}^{+\infty} g_{j}(\omega)\left\{e^{-\alpha_{j}(\omega) x} e^{i \omega\left[t-x / c_{j}(\omega)\right]} u(x)+e^{+\alpha_{j}(\omega) x} e^{i \omega\left[t+x / c_{j}(\omega)\right]} u(-x)\right\} d \omega .
$$

This impulse response is consequently the superposition of $J$ pairs of symmetrical decaying traveling waves starting from the excitation point and propagating in opposite directions. 
Thus, the general solution $y$ is given by the two-dimensional convolution:

$$
y(x, t)=\int_{-\infty}^{+\infty} \int_{-\infty}^{+\infty} f\left(x_{0}, t_{0}\right) h\left(x-x_{0}, t-t_{0}\right) d x_{0} d t_{0} .
$$

\section{Waveguide modeling of a region without sources}

Under the assumption that no sources exist in a region between $x=x_{\min }$ and $x=x_{\max }$, one can demonstrate that the vibration state is the sum of $J$ decaying traveling waves $y_{j}^{+}$in the increasing $x$ direction and of $J$ decaying traveling waves $y_{j}^{-}$in the decreasing $x$ direction:

$$
\begin{array}{r}
\forall x, x_{\min } \leqslant x \leqslant x_{\max }, \quad y(x, t)=\sum_{j=1}^{J} y_{j}^{+}(x, t)+\sum_{j=1}^{J} y_{j}^{-}(x, t), \\
\text { where } \widehat{y_{j}^{+}}(x, \omega)=e^{-i k_{j}(\omega)\left(x-x_{\min }\right)} \widehat{y_{j}^{+}}\left(x_{\min }, \omega\right) \\
\text { and } \widehat{y_{j}^{-}}(x, \omega)=e^{+i k_{j}(\omega)\left(x-x_{\max }\right)} \widehat{y_{j}^{-}}\left(x_{\max }, \omega\right) .
\end{array}
$$

This region can be modeled as $J$ waveguides in parallel which become $J$ digital waveguides ${ }^{\text {e.g. } 6,7}$ in discrete time. Each transfer function $e^{-i k_{j}(\omega) \Delta x}$ is generally designed as a delay line in series with a digital causal filter. After suitable digital filters have been found, efficient time-domain simulations can be made.

In the case of piano strings, the sources are located in the segment of hammer felt/string contact and at both ends. A block-diagram model is drawn in Fig. 1, including an observation point. This figure is a generalization of the model involved in Bensa et al. (Ref. 3, Fig. 2). Note that the boundary conditions characterizing the string terminations can be changed by only modifying one lumped filter at each end, apart from the waveguides modeling the portions of string without sources.

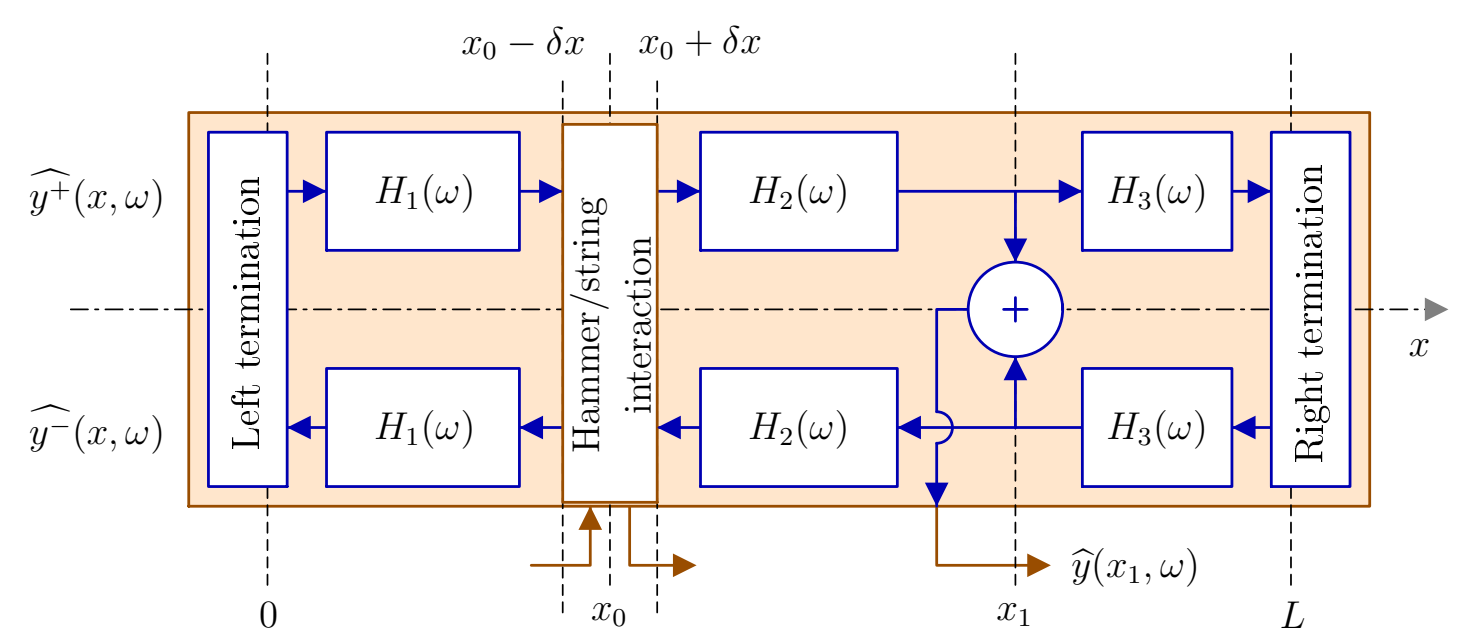

Figure 1: Block-diagram of the waveguide model of a piano string. The string segment interacting with the hammer felt is assumed to be $\left[x_{0}-\delta x ; x_{0}+\delta x\right] . x_{1}$ is the position of observation. $x=0$ is the capo d'astro bar position and $x=L$ is the bridge position. Each $H_{n}(\omega)$ is equivalent to $J$ delay line/filter blocks in parallel: $H_{1}(\omega)=\sum_{j=1}^{J} e^{-i k_{j}(\omega)\left(x_{0}-\delta x\right)}$, $H_{2}(\omega)=\sum_{j=1}^{J} e^{-i k_{j}(\omega)\left(x_{1}-x_{0}-\delta x\right)}$ and $H_{3}(\omega)=\sum_{j=1}^{J} e^{-i k_{j}(\omega)\left(L-x_{1}\right)}$ 


\section{THE FOUR WAVES IN A STIFF PIANO STRING}

In this section and the next one, we apply the principles given in the previous section to the one-dimensional wave equation introduced by Bensa et al. [Ref. 3, Eq. (6)]. The dispersion equation provides two pairs of decaying traveling waves which need a detailed analysis.

\section{A. The transfer function of a stiff piano string}

The transverse displacement $y$ is assumed to satisfy [Ref. 3, Eq. (6)]:

$$
\frac{\partial^{2} y}{\partial t^{2}}+2 b_{1} \frac{\partial y}{\partial t}-2 b_{2} \frac{\partial^{3} y}{\partial x^{2} \partial t}-c^{2} \frac{\partial^{2} y}{\partial x^{2}}+\kappa^{2} \frac{\partial^{4} y}{\partial x^{4}}=\frac{1}{\mu} f,
$$

where $b_{1}$ is the first coefficient of damping (due to viscosity of the air), $b_{2}$ the second damping coefficient (inner losses), $c=\sqrt{T / \mu}$ the transverse wave velocity of string ${ }^{4}, T$ the string tension, $\mu$ the linear mass density of string, $\kappa=c \sqrt{E I / T}$ a stiffness coefficient, $E$ the Young modulus, $I=S r^{2}$ the moment of inertia of the cross-section, $S$ the cross-section, and $r$ the radius of gyration.

In the wave-number/frequency domain, Eqs. (5) and (15) lead to the transfer function:

$$
H(k, \omega)=\frac{Y(k, \omega)}{F(k, \omega)}=\frac{1}{\mu\left[\left(-\omega^{2}+2 i \omega b_{1}\right)+\left(c^{2}+2 i \omega b_{2}\right) k^{2}+\kappa^{2} k^{4}\right]} .
$$

\section{B. Complex wave-numbers}

The two $(J=2)$ wave-numbers $k_{s}$ and $k_{d}$ satisfy:

$$
\begin{aligned}
& k_{s}^{2}(\omega)=\frac{c^{2}}{2 \kappa^{2}}\left[-1-i \frac{2 \omega}{c^{2}} b_{2}+\sqrt{1+\frac{4\left(\kappa^{2}-b_{2}^{2}\right) \omega^{2}}{c^{4}}+4 i \omega\left(\frac{1}{c^{2}} b_{2}-2 \frac{\kappa^{2}}{c^{4}} b_{1}\right)}\right], \\
& k_{d}^{2}(\omega)=\frac{c^{2}}{2 \kappa^{2}}\left[-1-i \frac{2 \omega}{c^{2}} b_{2}-\sqrt{1+\frac{4\left(\kappa^{2}-b_{2}^{2}\right) \omega^{2}}{c^{4}}+4 i \omega\left(\frac{1}{c^{2}} b_{2}-2 \frac{\kappa^{2}}{c^{4}} b_{1}\right)}\right] .
\end{aligned}
$$

These equations are similar to Eq. (11) in Ref. 3. The approaches, however, are different. In the modal approach ${ }^{3}$ $\beta_{+}$and $\beta_{-}$are real-valued functions of the complex frequency $s$. In the present wave approach $k_{s}$ and $k_{d}$ are the roots of the dispersion equation and are complex-valued functions of the common angular frequency $\omega$ (real number).

The assumptions [Ref. 3 Eqs. (24) and (25)]

$$
b_{1} b_{2} \ll c^{2} ; \quad b_{2}^{2} \ll \kappa^{2} \text { and } b_{1}^{2} \ll \omega^{2}
$$

lead to

$$
k_{s}(\omega) \simeq \frac{c}{\kappa} \sqrt{\frac{\xi}{2}}-i \frac{\kappa \omega}{c^{3}(1+\xi)} \sqrt{\frac{2}{\xi}}\left(b_{1}+\frac{c^{2} \xi}{2 \kappa^{2}} b_{2}\right)
$$

and

$$
k_{d}(\omega) \simeq \frac{1}{c^{3} \sqrt{2 \xi}(1+\xi)}\left(2 \omega^{2} b_{2}-c^{2} \xi b_{1}\right)-i \sqrt{\frac{2}{\xi}} \frac{\omega}{c},
$$

with

$$
\xi=-1+\sqrt{1+\frac{4 \kappa^{2}}{c^{4}} \omega^{2}} .
$$




\section{Waveguide modeling of portions of string without sources}

The first wave-number $k_{s}$ characterizes a weakly attenuated dispersive traveling wave similar to the one described by Bensa et al. [Ref. 3, Eq. (34)]. At low frequencies $\left[b_{1}^{2} \ll \omega^{2} \ll c^{4} /\left(4 \kappa^{2}\right)\right]$ the phase velocity is close to c. Its numerical values are between 160 and $420 \mathrm{~m} \cdot \mathrm{s}^{-1}$ for piano tones $\mathrm{C} 2$, C4, and $\mathrm{C} 7$ (Ref. 3, Table I). The attenuation per unit length $\alpha_{s} \simeq b_{1} / c$ is small : its numerical values $\left[10 \alpha_{s} / \log (10)\right]$ are less than $0.1 d B \cdot m^{-1}$.

The second wave-number $k_{d}$ represents a fast-decaying wave with a very high phase velocity. At low frequencies the phase velocity is approximately $c \kappa /\left(b_{2}-\kappa^{2} b_{1} / c^{2}\right)$. Its numerical values are in the range $2.6 \times 10^{5}$ to $1.62 \times 10^{6} \mathrm{~m} \cdot \mathrm{s}^{-1}$. The attenuation per unit length is $\alpha_{d} \simeq c / \kappa$. The numerical values $\left[10 \alpha_{d} / \log (10)\right]$ of $\alpha_{d}$ are greater than $1.1 \times 10^{3} \mathrm{~dB} \cdot \mathrm{m}^{-1}$. For any length $\Delta x$ greater than $1.8 \mathrm{~cm}, e^{-\alpha_{d} \Delta x}$ (the modulus of $e^{-i k_{d} \Delta x}$ ) is less than $10^{-2}$. This second wave is quasi-evanescent i.e., its phase velocity is almost infinite and its attenuation per unit length is high. It could represent the fact that a part of the energy of a hammer strike would instantly propagate along the stiff string around the contact region such as to avoid the formation of a sharp corner, as shown below and in accordance with Cremer $^{8}$.

Consequently, the waveguide model of a portion of string without sources will contain only a single delay line/filter (wave-number $k_{s}$ ) for each direction of propagation, provided that the portion of string is longer than a few centimeters. But the other wave characterized by $k_{d}$ cannot be neglected at the neighborhood of the excitation region, as shown in the following section.

\section{The impulse response of the string}

\section{A. Space/frequency domain}

In the space/frequency domain Eqs. (16 18), (6), and (9) imply that the Fourier transform of the impulse response is:

$$
\widehat{h}(x, \omega)=\mid \begin{array}{ll}
\widehat{h_{s}^{+}}(0, \omega) e^{-i k_{s}(\omega) x}+\widehat{h_{d}^{+}}(0, \omega) e^{-i k_{d}(\omega) x} & x>0 \\
\widehat{h_{s}^{+}}(0, \omega)+\widehat{h_{s}^{-}}(0, \omega)+\widehat{h_{d}^{+}}(0, \omega)+\widehat{h_{d}^{-}}(0, \omega) & x=0 \\
\widehat{h_{s}^{-}}(0, \omega) e^{+i k_{s}(\omega) x}+\widehat{h_{d}^{-}}(0, \omega) e^{+i k_{d}(\omega) x} & x<0
\end{array},
$$

where

$$
\begin{aligned}
& \widehat{h_{s}^{+}}(0, \omega)=\widehat{h_{s}^{-}}(0, \omega)=g_{s}(\omega)=\frac{-i}{2 \mu \kappa^{2}\left[k_{s}(\omega)^{2}-k_{d}(\omega)^{2}\right] k_{s}(\omega)} \\
& \widehat{h_{d}^{+}}(0, \omega)=\widehat{h_{d}^{-}}(0, \omega)=g_{d}(\omega)=\frac{i}{2 \mu \kappa^{2}\left[k_{s}(\omega)^{2}-k_{d}(\omega)^{2}\right] k_{d}(\omega)}
\end{aligned}
$$

The complex compliances $g_{s}(\omega)$ and $g_{d}(\omega)$ (unit: $m \cdot N^{-1}$ ) characterize the conversion of force to motion.

\section{B. Example of a $\mathrm{C} 2$ piano string}

The response $y$ of a $\mathrm{C} 2$ piano string (Ref. 3, Table I) to a downward Gaussian time impulse at a single point $x=0$ is given by Fig. 2. This response is very close to the negative of the impulse response $h$ in the bandwidth 0-20 kHz. The transverse displacement $y$ is the sum of two weakly attenuated traveling waves $y_{s}^{ \pm}$(wave-numbers $\pm k_{s}$ ) and of two fast-decaying waves $y_{d}^{ \pm}$(wave-numbers $\pm k_{d}$ ) going away symmetrically from the excitation point. In agreement with Refs. 8 and 2 (Section 2.18), "the bend is rounded appreciably by the stiffness of the string". The fast-decaying waves inhibit the sharp corner which could be generated by the weakly attenuated traveling waves, if the former were not considered. As shown in the previous section, only the weakly attenuated traveling waves exist far from the excitation point (more than $2 \mathrm{~cm}$ ), as well as in the neighborhood of the excitation point after $0.1 \mathrm{~ms}$. 


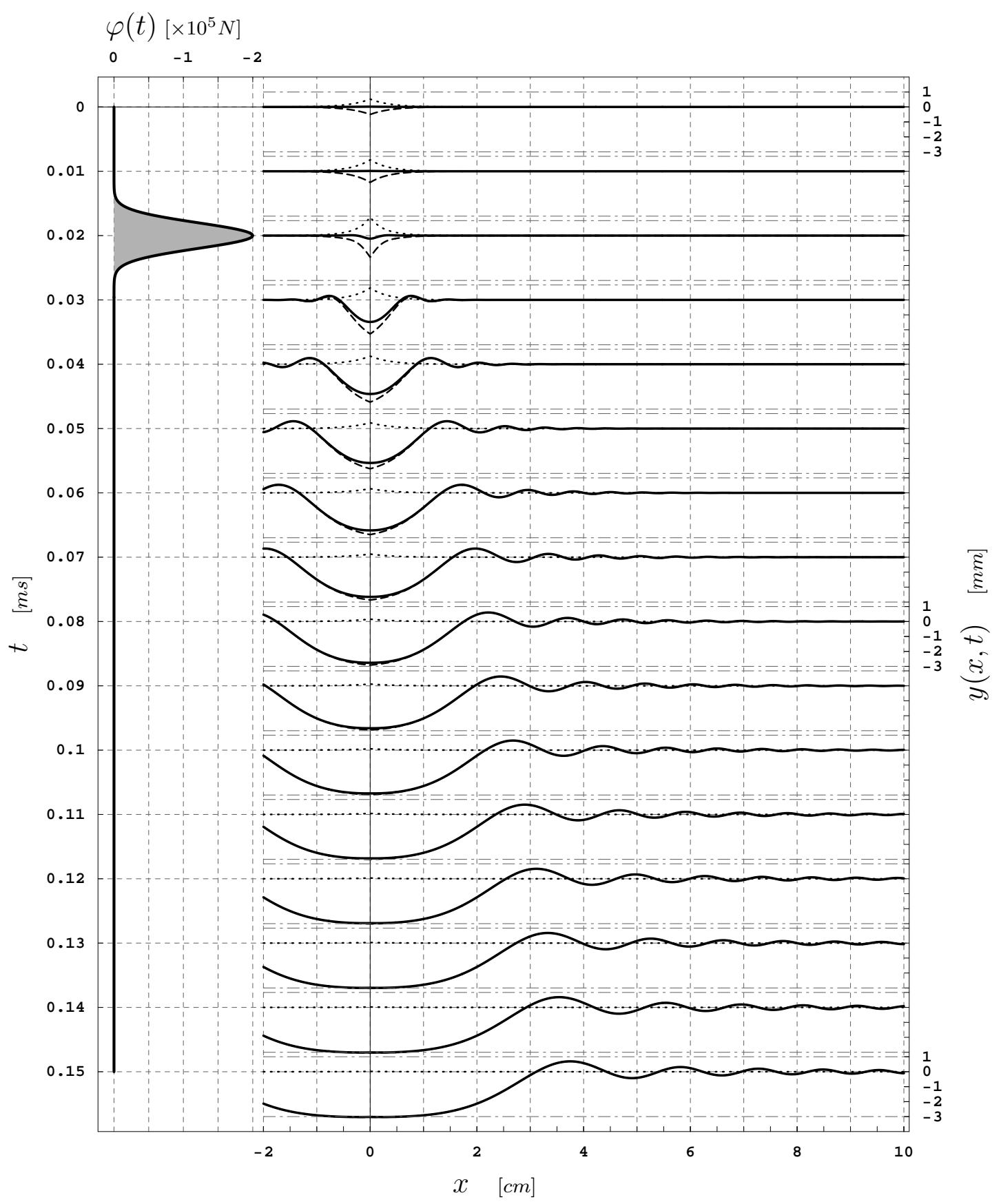

Figure 2: Response of a C2 piano string to a downward Gaussian impulse $f . f(x, t)=f_{0} \delta(x) \varphi(t)$ where $\varphi(t)=$ $-\lambda e^{-\pi \lambda^{2}(t-\tau)^{2}} . f_{0}=1 N, \lambda=2 \times 10^{5} \mathrm{~s}^{-1}$, and $\tau=0.02 \mathrm{~ms}$. The parameters of the $\mathrm{C} 2$ piano string are (Ref. 3 , Table I): $c=160.9 \mathrm{~m} \cdot \mathrm{s}^{-1}, \kappa=0.58 \mathrm{~m}^{2} \cdot \mathrm{s}^{-1}, b_{1}=0.25 \mathrm{~s}^{-1}$, and $b_{2}=7.5 \times 10^{-5} \mathrm{~m}^{2} \cdot \mathrm{s}^{-1}$. The response $y$ (plain line) is the sum of a weakly attenuated wave $y_{s}$ (dashed line) and of a quasi-evanescent wave $y_{d}$ (dotted line).

\section{Non-causal components of the causal impulse response}

An important additional point is that $y_{s}^{ \pm}$and $y_{d}^{ \pm}$are not causal responses whereas their sum is causal. A mathematical explanation can be found for this by focusing on the impulse response at the excitation point [Eq. (24)]. The signals $h_{s}^{ \pm}(0, t)$ and $h_{d}^{ \pm}(0, t)$ and the modulus of their Fourier transforms $g_{s}(\omega)=\widehat{h_{s}^{ \pm}}(0, \omega)$ and $g_{d}(\omega)=\widehat{h_{d}^{ \pm}}(0, \omega)$ are plotted in Fig. 3 (in the case of a C2 piano string). The latter signal $h_{d}^{ \pm}(0, t)$ is nearly an even function (with respect to $t$ ). The former signal $h_{s}^{ \pm}(0, t)$ is nearly the sum of a constant $[1 /(4 \mu c)]$ and of an odd function. 
(a)

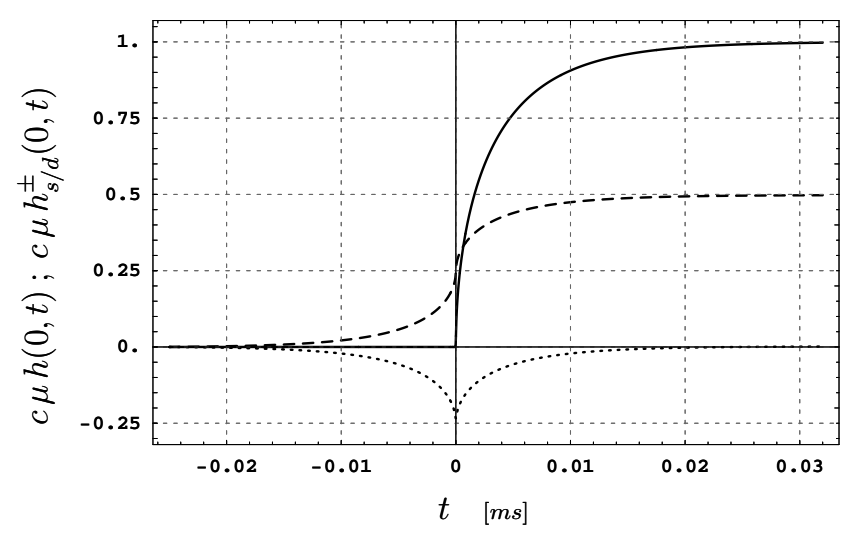

(b)

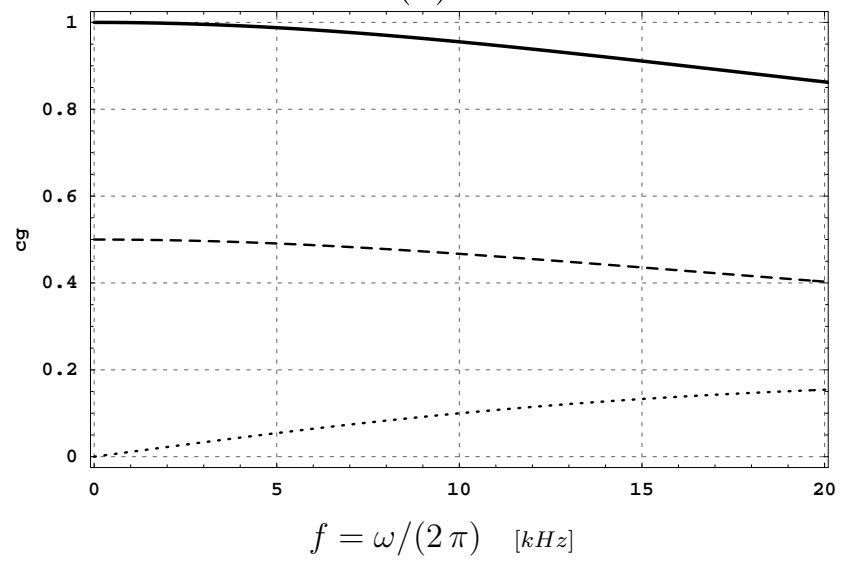

Figure 3: (a) Impulse responses of a $\mathrm{C} 2$ piano string and (b) the modulus of their Fourier transforms times the angular frequency. The compliance $g_{s}(\omega)=\widehat{h_{s}^{ \pm}}(0, \omega)$ is the Fourier transform of the signal $h_{s}^{ \pm}(0, t)$ (weakly attenuated waves, dashed line). $g_{d}(\omega)=\widehat{h_{d}^{ \pm}}(0, \omega)$ is the Fourier transform of $h_{d}^{ \pm}(0, t)$ (quasi-evanescent waves, dotted line). The impulse response $h(0, t)$ (plain line) is two times the sum of $h_{s}^{ \pm}(0, t)$ and $h_{d}^{ \pm}(0, t)$. Its Fourier transform is $\widehat{h}(0, \omega)=2\left[g_{s}(\omega)+g_{d}(\omega)\right]$.

This is natural because, on assuming that $b_{1}$ and $b_{2}$ are zero, and $\omega$ is greater than zero [see Eqs. (17)), (18), (22) , and (24)], the compliances become:

$$
g_{s}(\omega) \simeq \frac{i \kappa}{\mu c^{3} \sqrt{2 \xi}(1+\xi)} \quad \text { and } \quad g_{d}(\omega) \simeq \frac{-\sqrt{\xi}}{2 \mu \sqrt{2} c(1+\xi) \omega} .
$$

Consequently, the compliance $g_{s}$ is an imaginary-valued function and $g_{d}$ is a real-valued function. Because the signals $h_{s}^{ \pm}(0, t)$ and $h_{d}^{ \pm}(0, t)$ are real-valued, the former should be an odd function and the latter an even function. But the Fourier transform of $h_{s}^{ \pm}(0, t)$ has a singularity at $\omega=0$ and the limit of $h_{s}^{ \pm}(0, t)$, when $t$ tends to $-\infty$, has to be zero. This implies that a constant has to be added to $h_{s}^{ \pm}(0, t)$, giving an additional term $\pi \delta(\omega) /(2 \mu c)$ in its Fourier transform.

Applying the final value theorem to the signal $h_{s}^{ \pm}(0, t)$, the limit of $h_{s}^{ \pm}(0, t)$ with $t$ tending to infinity is $1 /(2 \mu c)$ (see Fig. 3). The final value of the impulse response $h(0, t)$ is similarly $1 /(\mu c)$. This last property is false if $b_{1}>0$ : all the signals tend slowly to zero when $t$ tends to infinity. Indeed, $h_{d}^{ \pm}(0, t)$ is close to zero for great values of $t$ and $h(0, t) \simeq h_{s}^{+}(0, t)+h_{s}^{-}(0, t)$.

In summary, the two waves $h_{s}^{ \pm}$and $h_{d}^{ \pm}$have to be considered together at the position $x=0$ to avoid nonphysical effects with respect to time. The conversion of force to motion is characterized by a single compliance $\widehat{h}(0, \omega)=2\left[g_{s}(\omega)+g_{d}(\omega)\right]$, which is plotted in Fig. 3 . 


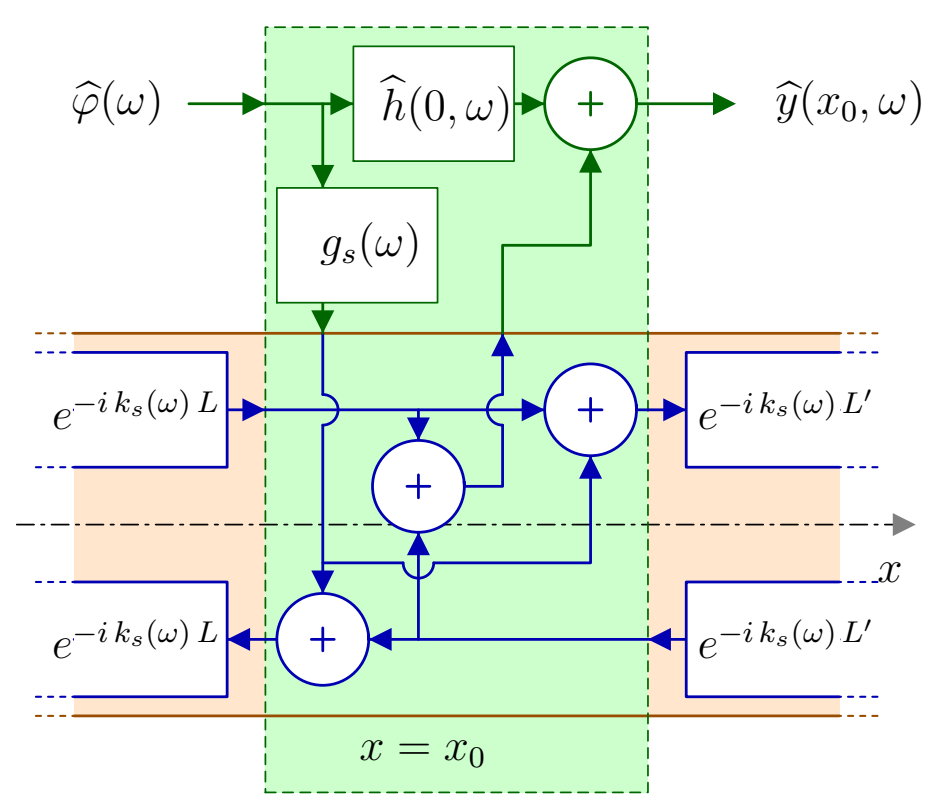

Figure 4: Block-diagram of a portion of string excited by a point force. This force $\varphi(t)$ is exerted by the hammer felt at the position $x=x_{0}$. The force distribution is assumed to be $f(x, t)=\varphi(t) \delta\left(x-x_{0}\right)$ for $x$ in the range $x_{0}-L$ to $x_{0}+L^{\prime}\left(L\right.$ and $L^{\prime}$ are both greater than a few centimeters).

This implies that if the force distribution $f(x, t)=\delta\left(x-x_{0}\right) \varphi(t)$ is assumed to represent a point-force exerted by the hammer felt at the position $x=x_{0}$, then the response $y\left(x_{0}, t\right)$ at the excitation point satisfies $\widehat{y}\left(x_{0}, \omega\right)=2\left[g_{s}(\omega)+g_{d}(\omega)\right] \widehat{\varphi}(\omega)$. This case is represented in Fig. 4 as a block-diagram. Similar block-diagrams are used for bowed strings (cf. e.g. the review article by $\mathrm{Smith}^{9}$, Fig. 14).

\section{CONCLUSION AND PROSPECTS}

The conclusion of this paper is that a complete mathematical treatment of the one-dimensional wave equation (15) carefully takes into account all the waves provided by the dispersion equation. Fast-decaying waves can be neglected in the waveguide modeling of portions of string without sources if they are long enough. However, they cannot accurately be neglected at the neighborhood of the excitation region (hammer) and of the fixation points (bridge, capo d'astro bar).

In particular if a point-force is exerted by the hammer felt on the string, the waveguide model proposed by Bensa et al. (Ref. 3, Fig. 2) needs to be complemented at the excitation point, as shown in Fig. 4 which summarizes the results of the last section. Note that a realistic excitation by the hammer is distributed over a certain width $^{4}$. This extension could be made by a spatial convolution. A future study may determine whether or not this correction would noticeably improve the quality of synthesized stiff-string sounds.

This paper is an attempt to extract as much information as possible from the physical model defined by a one-dimensional wave equation, in a waveguide modeling context and from a mathematical point of view. It is hoped that this study will help to improve future waveguide models of strings and to enhance the accuracy of efficient computational models using Digital Waveguides ${ }^{6,7,3}$, after a substantial effort to design suitable digital filters. 


\section{REFERENCES}

1 L. Hiller and P. Ruiz: "Synthesizing musical sounds by solving the wave equation for vibrating objects." J. Audio Eng. Soc. 19 462-472 (Part I) and 542-551 (Part II) (1971).

2 N. Fletcher and T. Rossing, The Physics of Musical Instruments (2nd ed., ISBN 0-387-98374-0, SpringerVerlag, New York, 1999).

3 J. Bensa, S. Bilbao, R. Kronland-Martinet, and J. O. Smith III: " The simulation of piano string vibration: from physical model to finite difference schemes and digital waveguides." J. Acoust. Soc. Am. 114(2) 1095-1107 (2003).

4 A. Chaigne and A. Askenfelt: "Numerical simulations of struck strings. I. A physical model for string using finite difference methods." J. Acoust. Soc. Am. 95(2) 1112-1118 (1994).

5 J.-M. Adrien: "The Missing Link: Modal Synthesis." in Representations of Musical Signals. MIT Press, 1991, 269-297.

6 J. O. Smith III: "Physical modeling using digital waveguides." Computer Music J. 16(4) 74-91 (1992).

7 J. O. Smith III: "Physical modeling synthesis update." Computer Music J. 20(2) 44-56 (1996).

8 L. Cremer, The Physics of Violin (translation by J.S. Allen, MIT Press, Cambridge, Massachusetts, 1984).

9 J. O. Smith III: "Virtual acoustic musical instruments: Review and update," J. New Music Research 33(3) 283-304 (2004), available online at http://ccrma.stanford.edu/ jos/jnmr/. 